\title{
Ho and NaD Line Variability in RY Tauri
}

\author{
P. Petrov ${ }^{1}$ and O. Vilhu ${ }^{2}$ \\ ${ }^{1}$ Crimean Astrophysical Observatory, 334413 Crimea, USSR \\ ${ }^{2}$ Observatory and Astrophysics Laboratory, University of Helsinki, \\ SF-00130 Helsinki, Finland
}

\begin{abstract}
Using high resolution spectroscopy of the T Tau star RY Tau in the regions of $\mathrm{H} \alpha$ and $\mathrm{Na} \mathrm{D}$ lines, we discovered line profile variability, which is interpreted by a hypothesis of stellar 'prominences': cool gas clouds are moving inside the stellar wind, ascending and descending on a time scale of a few days. These clouds are supposed to be controlled by the magnetic field of the star. The range of radial velocities of the clouds $(-100$ to $+100 \mathrm{~km} / \mathrm{s})$ is twice as large as the rotational velocity $(v \sin i)$ of the star. The form of variability of the $\mathrm{H} \alpha$ profile suggests the existence of two components: the (variable) stellar wind and the circumstellar disc.
\end{abstract}

\section{Introduction}

Since the pioneering work by Joy (1945), who first noticed the similarity between the emission spectrum of T Tau stars and the chromospheric spectrum of the Sun, the idea of the close physical connection between these two phenomena has been a leading one for many investigators. During the following half of the century much more data in favour of this concept were obtained: winds, spots, UV and X-ray radiation. In all these phenomena the magnetic field plays the major role.

Besides this pattern of solar-like activity, $T$ Tau stars exhibit another kind of spectroscopic and photometric variability, caused by gaseous and dust discs around the star (Bertout, 1988). The Sun also has a relic of the circumstellar disc (the solar system) but the solar activity is now controlled rather by internal than external forces. In $\mathrm{T}$ Tau stars the role of discs may be more important at certain stages of evolution.

The gas dynamics around the star can be studied by means of high resolution spectroscopy at certain wavelength regions. In this paper we discuss briefly the data of the Na D lines, published recently by one of us (Petrov, 1990). We also present new data of the $\mathrm{H} \alpha$ line variability in RY Tau. This star is a well known $\mathrm{T}$ Tau star with emission spectrum of moderate intensity and with underlying absorption spectrum of spectral type K1 IV-V $(v \sin i=53 \mathrm{~km} / \mathrm{s}$, Herbig and Bell, 1988). 

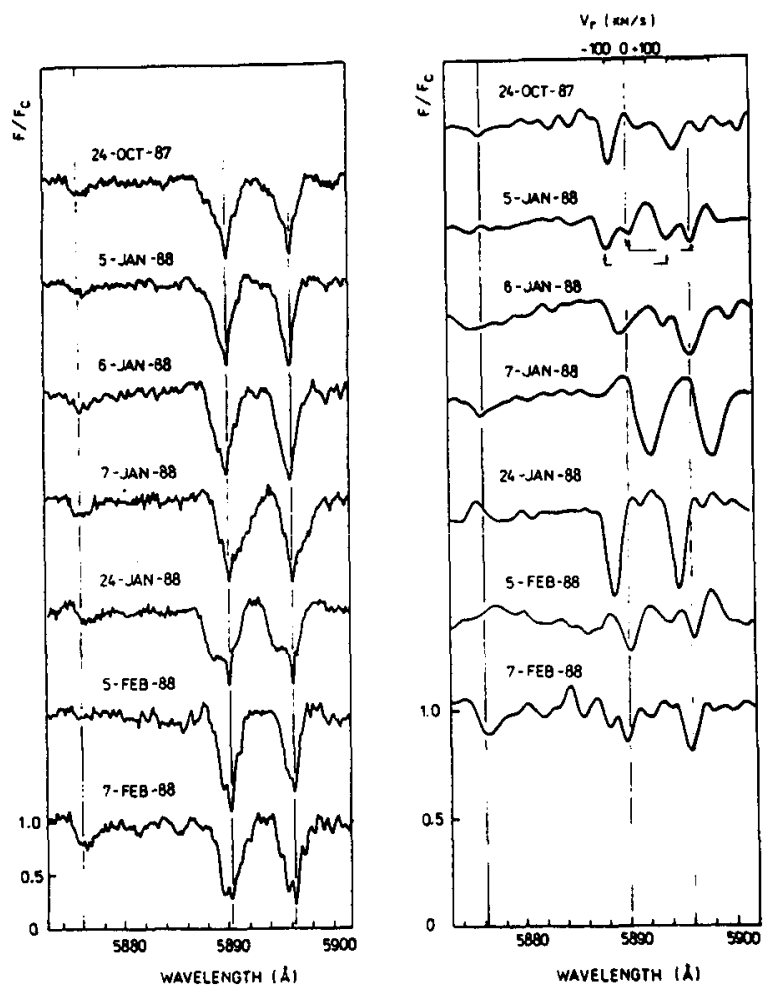

Fig. 1. a.) Spectra of RY Tau in the region of the Na D lines. b.) Differential spectra, derived by subtracting the average spectrum from the individual spectra (from Petrov, 1990).

\section{Variability of the $\mathrm{NaD}$ lines}

Spectroscopic observations of RY Tau in the regions of the Na D lines were carried out with the coudé spectrograph of the $2.6 \mathrm{~m}$ Shajn reflector of the Crimean Astrophysical Observatory, using the CCD camera of the Helsinki University. The spectral resolution was set by the entrance slit of 1 arc second, which corresponds to $0.37 \AA$ on the CCD. During the winter season of $1987 / 8821$ spectra of RY Tau were taken. A fragment of the data set, showing the most obvious variability of the profiles, is shown in Fig. 1a. Besides the photospheric components of the $\mathrm{Na}$ $\mathrm{D} 1$ and D2 absorptions (broadened to $53 \mathrm{~km} / \mathrm{s}$ by stellar rotation) and the narrow interstellar component, variable absorptions in the blue and red wings can be seen (see e.g. the red wing on 7-Jan-88 and the blue wing on 24-Jan-88). In order to extract these variable absorption components, we subtracted the average spectrum of RY Tau from each of the individual spectra. The differential (residual) spectra, smoothed to resolution of about $40 \mathrm{~km} / \mathrm{s}$, are shown in Fig. $1 \mathrm{~b}$.

The residual absorptions are variable both in intensity and radial velocity, moving within the range from -100 to $+100 \mathrm{~km} / \mathrm{s}$, which is twice as large as the $v \sin i$ of the star. This implies that the absorption does not originate from 
the stellar surface but more likely from the circumstellar environment. It could be clouds or streams of relatively cool gas inside the stellar wind. If the clouds were co-rotating with the star (trapped in the magnetic field loops as proposed by Cameron and Robinson (1989) for AB Dor), their radial velocities should not exceed the $v \sin i$-value of the star. In addition, if the clouds were orbiting the star, they must be on a very low Keplerian orbit with a period of about $8 \mathrm{~h}$, but the observations did not show any variability on a time scale of hours.

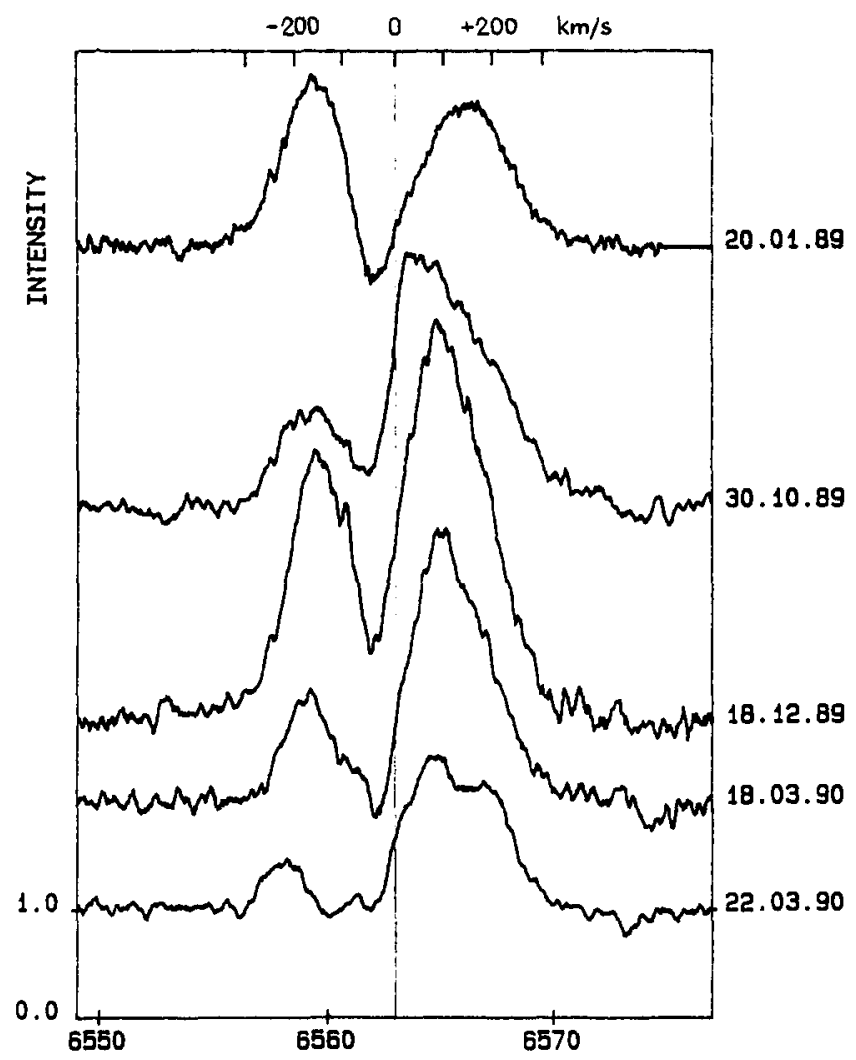

Fig. 2. Variations in the $\mathrm{H} \alpha$ line. All spectra are normalized to the continuum level. Intensity scale is shown for the lowest spectrum.

Consequently, we have to conclude, that the clouds are co-rotating with the star and have some radial velocity components. This is reminiscent of the phenomena of eruptive solar prominences and could be considered as being 'stellar prominences' on a young star. The general appearance of the residual spectra suggests that the temperature of the clouds might be in the range of $5000-7000 \mathrm{~K}$, which is also close to that of the solar prominences. We did not find any of the periodicity expected from rotational modulation. Probably the life time of these 'prominences' is not much longer than the rotational period of the star (about 5 days, Zaitseva et al., 1985). 


\section{Variability of the $H \alpha$ line}

In order to investigate the gas dynamics in the outer region of the circumstellar environment, we observed the $\mathrm{H} \alpha$ line between January 1989 and March 1990. The observations were a part of a more extended program of $\mathrm{T}$ Tau stars (in collaboration with Mark Giampapa).

Several typical $\mathrm{H} \alpha$ profiles are presented in Fig. 2, exhibiting the pattern of line variability. As in many other rapidly rotating T Tau stars, the $\mathrm{H} \alpha$ emission is broad and is split into two parts by the blue-shifted absorption. The wings of the emission extend from -300 to $+300 \mathrm{~km} / \mathrm{s}$. This is a very stable parameter of the line. Another stable parameter is the absorption at $-50 \mathrm{~km} / \mathrm{s}$. The range of the profile variability is shown in Fig. 3, where all the profiles are plotted together. At the bottom of Fig. 3, the dispersion of line intensities is drawn. This dispersion function reveals that the most variable part of the $\mathrm{H} \alpha$ line is located at +50 $\mathrm{km} / \mathrm{s}$. This does not fit exactly with the red emission peak. In the red wing of the emission, the dispersion drops to zero already by the half maximum of the line, i.e. the red emission wing is not variable. The blue peak is often disturbed by a broad absorption centered at $-150 \mathrm{~km} / \mathrm{s}$.

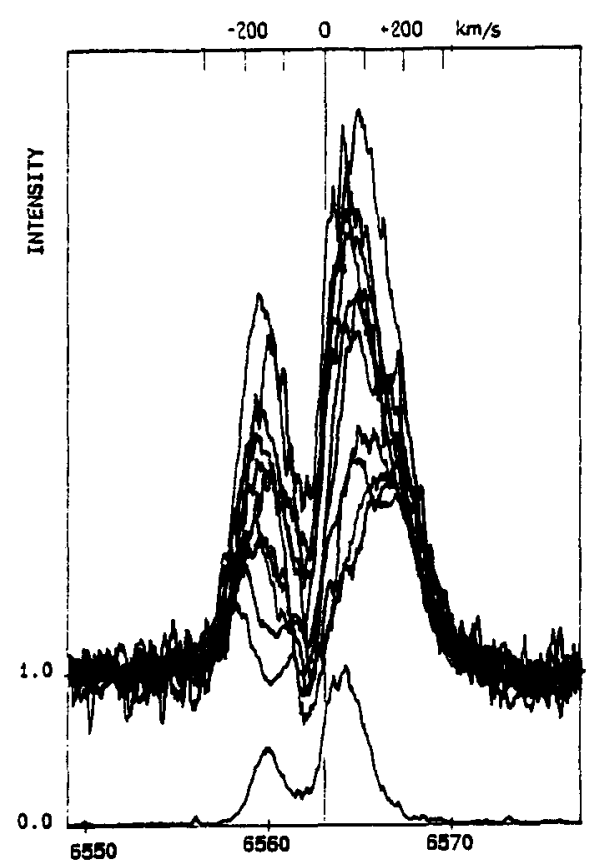

Fig. 3. Range of variability in the $\mathrm{H} \alpha$ profile. The lower curve is the dispersion of intensities.

A qualitative interpretation could be the following. The stable absorption at $-50 \mathrm{~km} / \mathrm{s}$ is formed at the most extended outflowing part of the wind, while the highly variable broad absorption at $-150 \mathrm{~km} / \mathrm{s}$ originates from the innermost part 
of the wind. It could be produced by the same streams or clouds ('prominences') that we observed in the Na D lines. Fig. 4 shows this effect both in the $\mathrm{H} \alpha$ and $\mathrm{Na} \mathrm{D}$ lines observed during the same night.
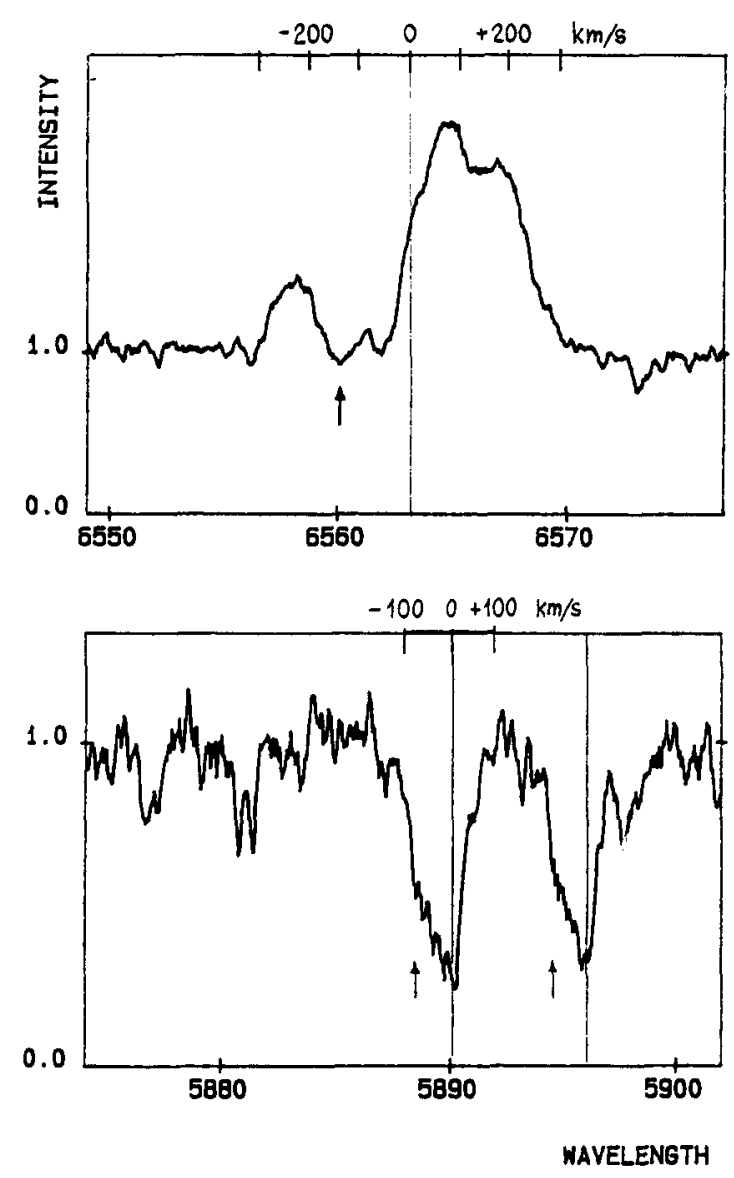

Fig. 4. Appearance of the broad blue shifted absorption in the $\mathrm{H} \alpha$ and $\mathrm{Na} \mathrm{D}$ lines. Both spectra were taken on March 22, 1990.

The co-existence of the broad stable wings and highly variable emission peaks suggests, that we observe two different components: one (stable) from the disk and another (variable) from the structured wind (see Fig. 5). 


\section{Conclusions}

Our observations of the $\mathrm{Na} \mathrm{D}$ and $\mathrm{H} \alpha$ line variability in RY Tau reveal the complicated structure of the circumstellar gas environment. Closer to the star we observed 'stellar prominences', while the extended parts of the gas envelope can be described in terms of a stellar wind of variable power. The structure of the $\mathrm{H} \alpha$ line also suggests the existence of a disc around the star.

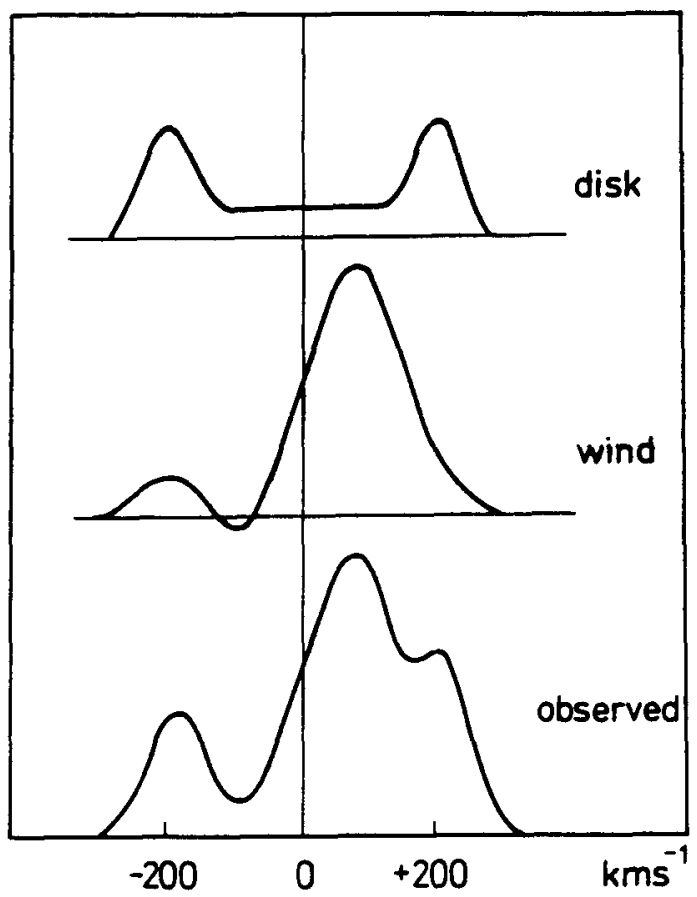

Fig. 5. A schematic explanation of the observed $\mathrm{H} \alpha$-profile as a sum of the disk and wind.

\section{References}

Bertout, C.: 1988, in A.K. Dupree and M.T.V.T. Lago (eds.), Formation and Evolution of Low Mass Stars, NATO ASI Series, Kluwer Academic Publishers, Dordrecht, Holland Cameron, A.C., Robinson, R.D.: 1989, Monthly Notices Roy. Astron. Soc. 236, 57

Joy, A.H.: 1945, Astrophys. J. 102, 168

Herbig, G.H., Bell, K.R.: 1988, Lick Observatory Bull. No. 1111.

Petrov, P.P.: 1990, Astrophysics and Space Sci. 169, 61

Zaitseva, G.V., Kolotilov, E.A., Petrov, P.P., Tarasov, E.A., Shenavrin, V.I., Shcherbakov, A.G.: 1985, Soviet Astron. Letters 11, 271 